\title{
Archéopages
}

Archéopages

Archéologie et société

Hors-série 2 | 2010

Archéologie sans frontières

\section{Paharpur, ou comment protéger et restaurer un site dégradé grâce à l'expertise archéologique}

Jean-Yves Breuil et Sandrine Gill

\section{CpenEdition}

1 Journals

Édition électronique

URL : https://journals.openedition.org/archeopages/741

DOI : 10.4000/archeopages.741

ISSN : 2269-9872

Éditeur

INRAP - Institut national de recherches archéologiques préventives

Édition imprimée

Date de publication : 1 octobre 2010

Pagination : 19-25

ISSN : 1622-8545

\section{Référence électronique}

Jean-Yves Breuil et Sandrine Gill, «Paharpur, ou comment protéger et restaurer un site dégradé grâce à l'expertise archéologique », Archéopages [En ligne], Hors-série 2 | 2010, mis en ligne le 01 octobre 2010, consulté le 23 février 2023. URL : http://journals.openedition.org/archeopages/741 ; DOI : https://doi.org/10.4000/archeopages.741 
sur une courte durée, accommodation de la recherche et des aménagements et travail en multi partenariat. C'est l'ensemble de ce défi qu'une collaboration extérieure à l'administration indienne, celle des archéologues de l'Inrap par exemple, pourrait relever.

GILL S., 2010, "Celestial women in a ring around the Buddhist stūpa: the case of Sanghol », in RAY H.P. (ÉD.), Sanghol and the Archaeology of Punjab, New Delhi, p. 129-151.

Gupta S. P. (ÉD.), 1985, Kushana sculptures from Sanghol $\left(1^{s t}-2^{\text {nd }} A D\right)$. A recent discovery, National Museum, New Delhi.

Indian Archaeology - A Review, «Exploration and Excavations: excavation at Sanghol, district Ludhiana », 1968-69, 1969-70, 1970-71, 1971-72, 1972-73, 1977-78, 1980-81,1984-85, 1985-86, 1986-87, 1987-88, 1988-89, 1989-90.

Margabandhu C., Gaur G. S., 1987, « Some fresh evidence from Sanghol excavations, $1986 »$, Purāttna, n 16 , New Delhi, p. 74-78

Margabandhu C., Gaur G. S., 1988, «Sanghol excavations 1987: some new evidences ", Purātattva - Bulletin of the Indian Archaeological Society, ${ }^{\circ}$ 17, New Delhi, p. 1-5.

Michon D., 2010, « The Excavation at Sanghol in Context », in Ray H. P. (ÉD.) Sanghol and the Archaeology of Punjab, New Delhi, p. 69-106.

Ray H.P.(ÉD.), 2010, Sanghol and the Archaeology of Punjab, New Delhi. Sharma G. B., 1986, Coins, seals and sealings from Sanghol, Department of Cultural Affairs, Archaeology and Museums, Punjab.

\section{Paharpur, ou comment protéger et restaurer un site dégradé grâce à l'expertise archéologique}

\author{
Jean-Yves Breuil \\ Inrap \\ Sandrine Gill \\ Creops
}

$\mathrm{L}$ e monastère bouddhique de Paharpur, aujourd'hui le Bangladesh, a été fondé à la fin du viII ${ }^{\mathrm{e}}$ siècle. Centre intellectuel de renom du bouddhisme d'Inde orientale d'époque Pāla, considéré à son apogée comme le plus grand monastère bouddhique du sous-continent indien, il possède en son centre un imposant monument de culte cruciforme, dont le modèle fut diffusé dans toute l'Asie du Sud-Est, et un ensemble exceptionnel de 2800 plaques de terre cuite figurées, disposées en frise sur les différentes terrasses du monument. Ces principaux critères lui doivent de figurer depuis 1985 sur la liste du Patrimoine mondial de l'Unesco. Cette mise en lumière internationale s'est malheureusement accompagnée d'une campagne de restauration tout à fait excessive qui a fortement dégradé l'authenticité d'un site déjà fragilisé par des décennies d'exposition à des contraintes environnementales fortes et à un vandalisme récurrent. Plusieurs missions initiées par le Centre du Patrimoine mondial de l'Unesco ont tenté de freiner ce processus et d'améliorer la gestion archéologique de ce site bouddhique très réputé.

Un grand monastère Pāla. Le site de Paharpur se situe près du village de Jaipurhat dans l'actuel district de Naogaon, dans le nord-ouest du Bangladesh, à 80 km au nord-est de Rajshahi et des rives du Gange [Fig.1]. Il abrite les vestiges d'un immense monastère bouddhique, dont la taille exceptionnelle le distingue de la nébuleuse des sites bouddhiques actifs à l'époque Pāla (VIII ${ }^{\mathrm{e}}-\mathrm{XII}^{\mathrm{e}}$ siècles). Au centre du monastère trône un monument de culte imposant [Fig.2], interprété comme une combinaison entre un temple et un stupa, monument bouddhique par excellence.

Paharpur, l'ancienne Somapura Mahāvihāra, fut fondé par le second empereur de la dynastie indienne Pāla, Dharmapāla, qui régna entre 770 et 810. À cette époque, la région de Paharpur, le Varendra, comme toute l'Inde orientale, était sous la domination de la dynastie Pāla dont les dirigeants étaient de fervents défenseurs du bouddhisme, sans qu'ils en aient fait pour autant une religion d'État. Ils parrainèrent la construction de nombreux stupas et vihara (monastères), centres d'apprentissage du bouddhisme du Grand Véhicule (Mahāyāna). De grands sites bouddhiques connurent alors un développement important pendant plusieurs siècles, tels Mainamati au sud-est du Bangladesh ou Nalanda au Bihar indien. $\mathrm{Au} \mathrm{XII}{ }^{\mathrm{e}}$ siècle, un moine du monastère de Nalanda, en pérégrination au nord du Bengale, décrit Paharpur comme « un festin exceptionnel pour les yeux du monde ». La renommée du monastère de Paharpur est attestée dans les sources indiennes, tibétaines et chinoises. Ce site, qui apparaît de nos jours isolé dans la campagne bangladaise, était à l'origine entouré par un important réseau de sites bouddhiques, comme en témoignent la présence, dans un rayon de $40 \mathrm{~km}$, de plusieurs monastères récemment redécouverts : Halud vihāra, Jagdal vihāra, Jagjivanpur, Vasu Bihar et Bihar (Breuil, Gill, 2007). Laissé à l'abandon au moment de la conquête islamique au début du XIII ${ }^{\mathrm{e}}$ siècle, le site de Paharpur fut redécouvert au début du XIX ${ }^{\mathrm{e}}$ siècle par le Britannique Buchanan Hamilton. Les premières fouilles commencèrent en 1923 sous l'égide de l'Archaeological Survey of India et se déroulèrent pendant une dizaine d'années jusqu'en 1934, principalement sous la direction de K. N. Dikshit (Dikshit, 1938). Les fouilles furent accompagnées d'une campagne de restauration de certaines parties du monument. À la suite de la demande du gouvernement du Bangladesh, Paharpur fut classé sur la liste du Patrimoine mondial culturel en 1985, en même temps que la mosquée de Bagerhat (Khalifatabad) datée $\mathrm{du} \mathrm{Xv}^{\mathrm{e}}$ siècle et située dans le sud-ouest du pays.2. Depuis les années 1980, des fouilles de petite superficie sont irrégulièrement menées, par le Département d'Archéologie du Bangladesh, dans la cour et les cellules du monastère.

Le site se compose d'un monastère principal construit en briques, de plan carré (environ 280 m de côté), composé de 177 cellules de moine ouvrant sur une cour au centre de laquelle est érigé le monument de culte cruciforme (dont la partie préservée mesure encore $22 \mathrm{~m}$ de hauteur). Un certain nombre de structures annexes sont bâties dans la cour et à l'extérieur du monastère [Fig.1].

Les investigations récentes ont confirmé l'existence de quatre états de construction et reconstruction, tant pour le monastère principal 


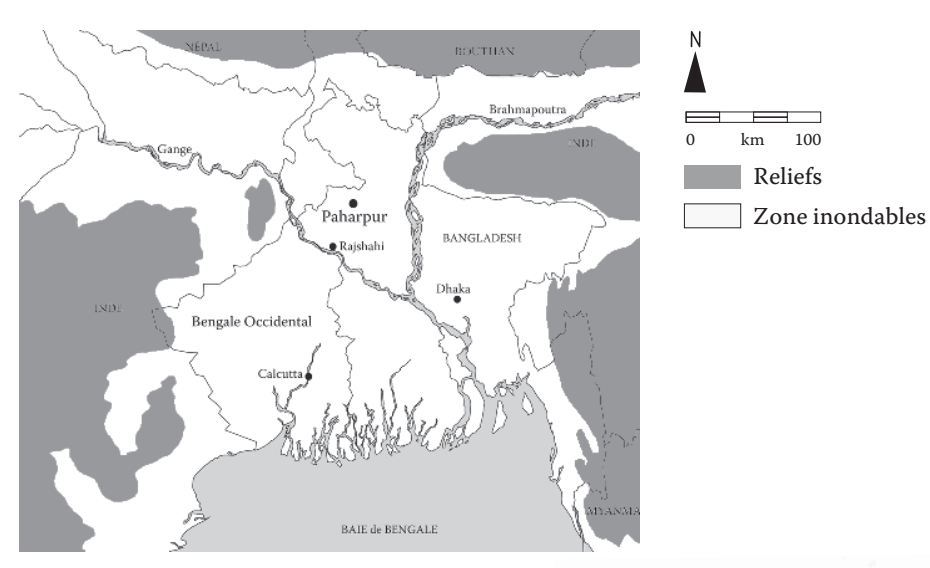

ลี

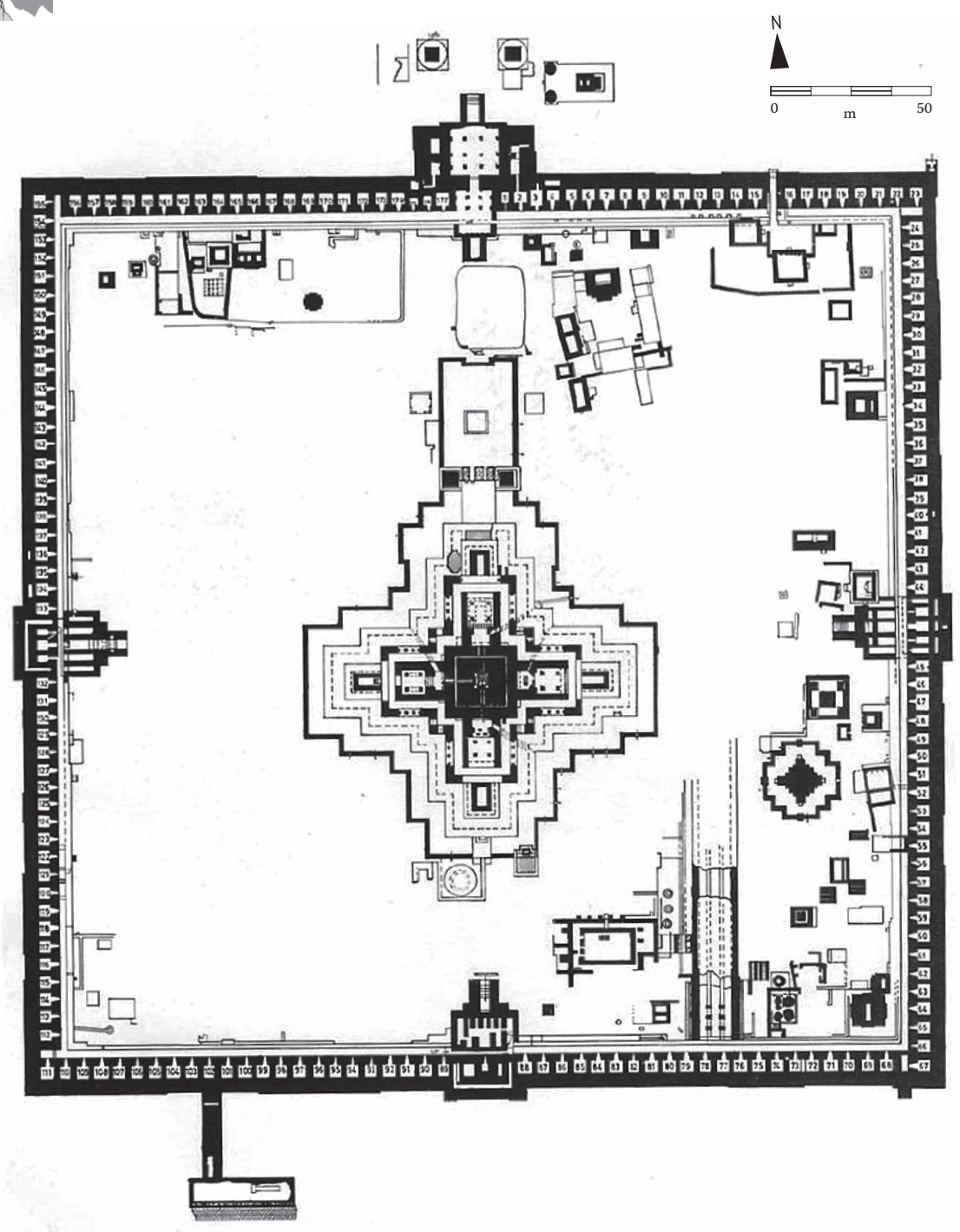


que pour le temple central. Elles ont aussi montré l'existence d'une occupation antérieure au monastère sans qu'il soit possible d'en préciser la nature ni la datation exacte.

À l'origine, le visiteur entrait dans le monastère par un portail monumental situé au nord puis, après avoir dépassé un ensemble de structures votives, il pénétrait dans un vestibule précédant l'escalier imposant du monument central. Organisé autour d'une cella centrale aveugle de forme carrée, entouré par des salles à piliers (mandapa) munies d'antichambres sur les quatre côtés, qui correspondent aux directions cardinales, le monument central forme une sorte de mandapa tridimensionnel. Il s'élève sur trois niveaux de terrasses qui permettent aux fidèles d'accomplir le rituel de la circumambulation sur le pradak ināpatha (chemin) jusqu'au sommet du monument dont la superstructure est perdue. La forme du temple central a été réutilisée et interprétée par la suite dans l'architecture bouddhique au Tibet, et surtout en Asie du Sud-Est, notamment en Birmanie, au Cambodge et à Java.

La base du monument [Fig.3], aujourd'hui en partie enterrée, est décorée par 63 sculptures en pierre représentant des divinités hindoues réemployées de temples plus anciens et par deux rangées de plaques en terre cuite. Cette intégration d'images de divinités hindoues dans un monument bouddhique peut trouver différentes explications : le goût éclectique du fondateur du temple, Dharmapāla ; la récupération d'éléments décoratifs en pierre, très précieux dans une région dépourvue de ressources lithiques ; l'intégration de divinités hindoues à la périphérie d'un diagramme mystique (mandala) formé par le plan concentrique du temple, etc. La disposition des sculptures autour d'un réservoir rituel à la base du monument indique qu'elles étaient prévues dans le programme initial d'architecture. Deux rangs de plaques en terre cuite décorent la terrasse supérieure ; elles portent des scènes de la vie quotidienne, du folklore local, des êtres fantastiques, des animaux [Fig.4], plus rarement des divinités bouddhistes et hindoues ; quelques scènes sont tirées des grandes épopées indiennes comme le Mahābhärata ou le Rāmāyana et des récits évoquant les vies antérieures du Bouddha. Ces plaques font preuve d'une aisance exceptionnelle dans le modelage des figures, qui transgressent souvent les limites du cadre et sont beaucoup plus libres dans leur expression que les sculptures contemporaines de style Pāla. L'ensemble des frises faisaient partie du décor architectural destiné à ponctuer et à rythmer le parcours du fidèle, sans pour autant se substituer aux images de culte. Le programme iconographique reste difficile à comprendre car, sur les 2800 plaques de terre cuite inventoriées dans les années 1930, seules 2 ooo étaient $i n$ situ, une situation qui a encore empiré ces dernières années avec seulement 500 plaques originelles recensées sur le monument. La disposition, de certaines plaques à l'envers ou de travers, a contribué à l'idée que l'ensemble était le résultat de remaniements multiples. Il apparaît toutefois que certaines séquences de plaques portent indubitablement les traces d'une composition basée sur le principe de la répétition du même motif, de l'alternance et de l'opposition qui créent un rythme et un mouvement sous-jacent au mode de narration. Ainsi plusieurs ensembles de plaques représentent, dans un style relevé, des scènes de chasse par la juxtaposition de figures individuelles liées entre elles par une même thématique : animaux sauvages et figures armées. Ce type de narration, destiné à accompagner le mouvement rituel du fidèle, est typique des temples indiens, qu'ils soient bouddhiques ou hindous. Aucune image de culte n'a été trouvée in situ dans les chapelles du temple mais le buste en bronze d'un bouddha colossal (130 cm préservés) découvert dans une cellule du monastère en 1982 a peut-être appartenu au temple central.

Dans le monastère, les bâtiments centraux des ailes est, sud et ouest ont été utilisés comme des lieux saints annexes. Le responsable ou le moine aîné du monastère vivait sans doute dans les cellules à l'est de l'entrée principale, là où un riche mobilier a été retrouvé (monnaies, sceaux, perles, vaisselle en bronze, etc.). Les cellules des moines, ouvertes sur la grande cour centrale, sont alignées à la suite les unes des autres. Chaque cellule a une superficie moyenne de 4,20 $\mathrm{m}^{2}$ et possède une arrière-chambre rectangulaire.

De nombreuses structures ont été identifiées dans la cour : au milieu des petits sanctuaires et autres stupas votifs, parmi lesquels une réplique du temple central, plusieurs édifices, situés dans la partie sud-est de la cour, sont liés à la vie en commun des moines (cuisine, réfectoire, puits). Un système complexe de drainage a été aménagé sur le monument central et dans l'ensemble de la cour, démontrant l'acuité du problème de la gestion des eaux de ruissellement dès la création du monastère.

À l'extérieur du monastère sont bâties des structures servant aux ablutions et un $g h a \bar{t}$ (plusieurs marches) conduit à l'ancien lit d'une rivière. À 270 m plus à l'est, il existe un temple plus tardif, localement connu comme « Satyabir Bhita », qui est mentionné dans une inscription d'un moine de Nalanda au début du XII ${ }^{\mathrm{e}}$ siècle. Consacré à la déesse Tārā, il est entouré par 132 structures votives et un mur d'enceinte.

La densité des structures et la richesse iconographique tendent à montrer que l'organisation du culte à Paharpur était complexe. Le site a été un centre d'attraction et surtout de propagation de la culture indienne et bouddhiste vers le Tibet et l'Asie du Sud-Est.

\section{Problèmes de conservation et restauration excessive}

Dès sa mise au jour dans les années 1930, le site de Paharpur, et en particulier le monument de culte central, a été confronté à de cruciaux problèmes de conservation liés aux très fortes pluies de mousson de cette région et à la remontée induite de la nappe phréatique. À l'issue de la fouille, pour que l'édifice soit stabilisé, le soubassement et le niveau inférieur de la cour environnant le monument ont été enterrés, sans la mise en place d'un drainage adéquat. 


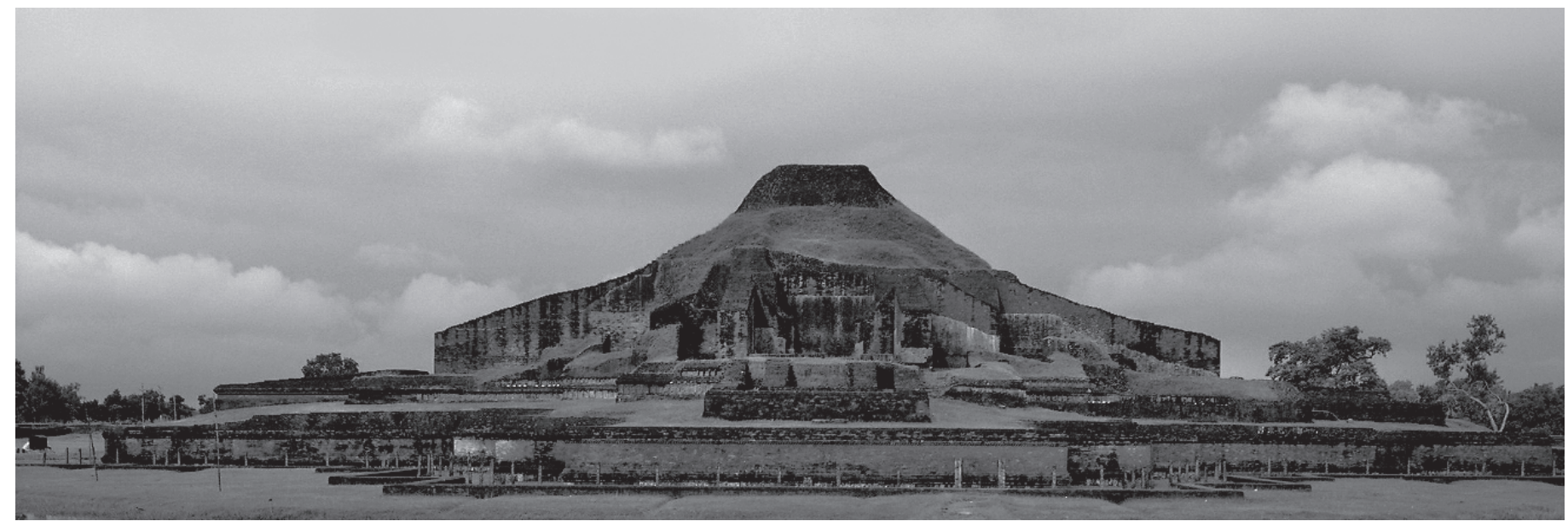

ก
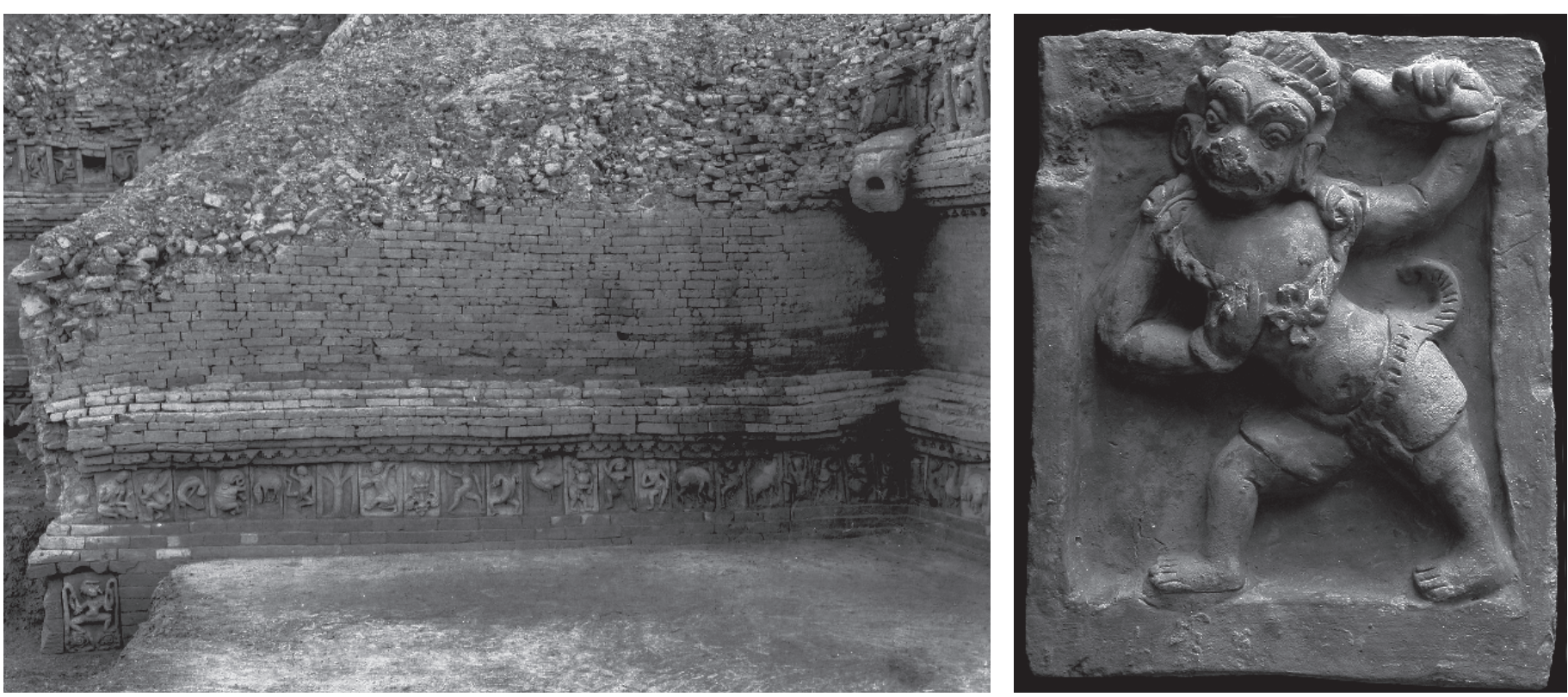

[Fig.2] Le monument de culte central de Paharpur, après sa dernière restauration, vu de l'est (octobre 2002).
[Fig.3] Dégagement du premier niveau du temple central de Paharpur durant les fouilles des années 1930.
[Fig.4] Plaque de terre cuite portant

la représentation d'un singe. 
Soixante années d'exposition à des précipitations très importantes ont continué de fragiliser le monument. La surface des briques et les plaques de terre cuite ont été affectées par l'attaque de sulfate, la présence de sels dans l'eau et le développement de mousses. À cet environnement climatique défavorable se sont ajoutés les dommages liés à une mauvaise gestion du site, qui a conduit en particulier à un vandalisme répété des vestiges et à plusieurs vols de plaques décoratives en terre cuite.

Pour pallier ces dégradations, le département de l'Archéologie du Bangladesh, avec l'appui technique et financier de l'Unesco, a entrepris pendant des années un certain nombre de travaux visant à trouver des solutions pour le drainage et la préservation du monument.

Mais les travaux de restauration du monument, débutés en 1991 et terminés en 2002, ont eu des conséquences désastreuses, à un degré tel que le comité annuel du Centre du Patrimoine mondial de l'Unesco, réuni en juin 2002, a dépêché une mission d'expertise (R. Engelheart, J.-Y. Breuil et S. Gill) afin de mesurer l'état de dégradation du site et de proposer d'éventuelles mesures d'amélioration. De fait, conduite par des ingénieurs peu soucieux de la réalité historique, la « restauration » a quasientièrement reconstruit le monument en brique avec du matériau neuf, en gommant toutes les « anomalies » architecturales et a remplacé les plaques de terre cuite originelles par de prétendues " répliques », imitations lointaines des plaques de style Pāla, effectuées par des artisans locaux. Au cours de ces travaux, beaucoup d'éléments architecturaux d'origine et des plaques de terre cuite ont été endommagés ou perdus. Les transformations dues à ces travaux de restauration ont sérieusement altéré et changé le degré d'authenticité et d'intégrité du monument, à un point tel qu'il est devenu difficile, pour les historiens et pour le public, de « lire » la forme et la fonction originelles du monument et de comprendre son histoire archéologique.

Une authenticité à reconstituer. Face à cette situation, et pour rendre au site de Paharpur une certaine authenticité, les premières recommandations ont été :

- la mise en œuvre d'un moratoire, c'est-à-dire un arrêt total des travaux de restauration;

- la mise en place d'un inventaire systématique de toutes les plaques de terre cuite décoratives conservées dans les réserves du musée et celles encore in situ, avec l'objectif d'éditer un catalogue complet;

- une amélioration du système de surveillance et d'information du site;

- une étude du drainage pendant une année, par un hydrologue, en étroite collaboration avec un archéologue.

Par ailleurs, un travail d'exploitation d'anciennes photographies du site a été engagé. Un ensemble remarquable de 1700 photographies documente en effet chaque étape de la découverte du site jusqu'à sa première restauration, dans les années 1920-30.
Quinze pour cent seulement de cette documentation fut publiée en 1938 dans la monographie du site par K. N. Dikshit. De fait, ces plaques de verre, numérisées grâce à l'ambassade de France au Bangladesh et documentées par l'équipe archéologique française de Mahasthan (nordouest du Bangladesh), constituent un outil indispensable à la compréhension du site (Breuil, Gill, 2005).

Une seconde mission, en février 2003, a permis le suivi des recommandations, la planification de formations techniques et l'organisation d'un séminaire de travail international. Celui-ci portait sur « l'élaboration d'une stratégie de recherche archéologique pour le site de Paharpur et ses environs » et s'est tenu en mars 2004 à Dhaka. Rassemblant 70 spécialistes, il a permis de rappeler le contexte et la valeur historique du site, d'appréhender Paharpur dans ses différentes composantes, environnementale, économique, technique, touristique, religieuse, scientifique, et de définir les priorités pour améliorer la gestion du site et la conservation du monument et des mobiliers. À la fin de l'année 2004, deux formations conduites par A. Prieur (CNRS, Lyon) et K. K. Jaïn (National Research Laboratory for Conservation of Cultural Property, Lucknow, Inde) et adressées en particulier à l'ensemble des personnels du site ont été consacrées à la gestion, la manipulation, le stockage, le nettoyage et la conservation des plaques de terre cuite. Une base de données sur ces plaques a été créée à l'issue de ces formations.

La situation n'a pas pour autant été renversée. L'authenticité du monument de culte n'a pas été restaurée et la lecture historique du site demeure problématique. De nos jours, le visiteur a une vision erronée et très simpliste de Paharpur : le monument se dresse dans une cour quasiment vide; il a l'aspect d'une construction neuve où aucune phase de reconstruction et de réaménagement n'apparaît ; toutes les aspérités de l'histoire ont été gommées. Les pseudo-répliques ne reflètent aucunement le style original des plaques figuratives. Les frises de terre cuite sur la base du monument, originellement à hauteur des yeux, se situent au niveau du sol ; les sculptures en pierre du soubassement sont soit exposées dans le musée du site, soit encore enfouies in situ. La perception du site est également faussée par l'entrée actuelle du monastère à l'est (plutôt qu'au nord) et par l'ensemble de constructions modernes entre le monastère et le temple de Tārā. Ces deux principaux monuments du site, en lien l'un avec l'autre, semblent indépendants dans la configuration actuelle.

L'exposition d'un tel site à un niveau international, loin de l'effet protecteur escompté, a accéléré dans un premier temps som processus de dégradation (pillage, restauration grossière). Cependant les diverses missions ont permis d'amorcer un lent processus d'amélioration dans la gestion du site, en particulier dans l'attention portée aux plaques figuratives, véritable trésor dans l'art de la terre cuite du Bengale. Elles ont permis de fédérer 


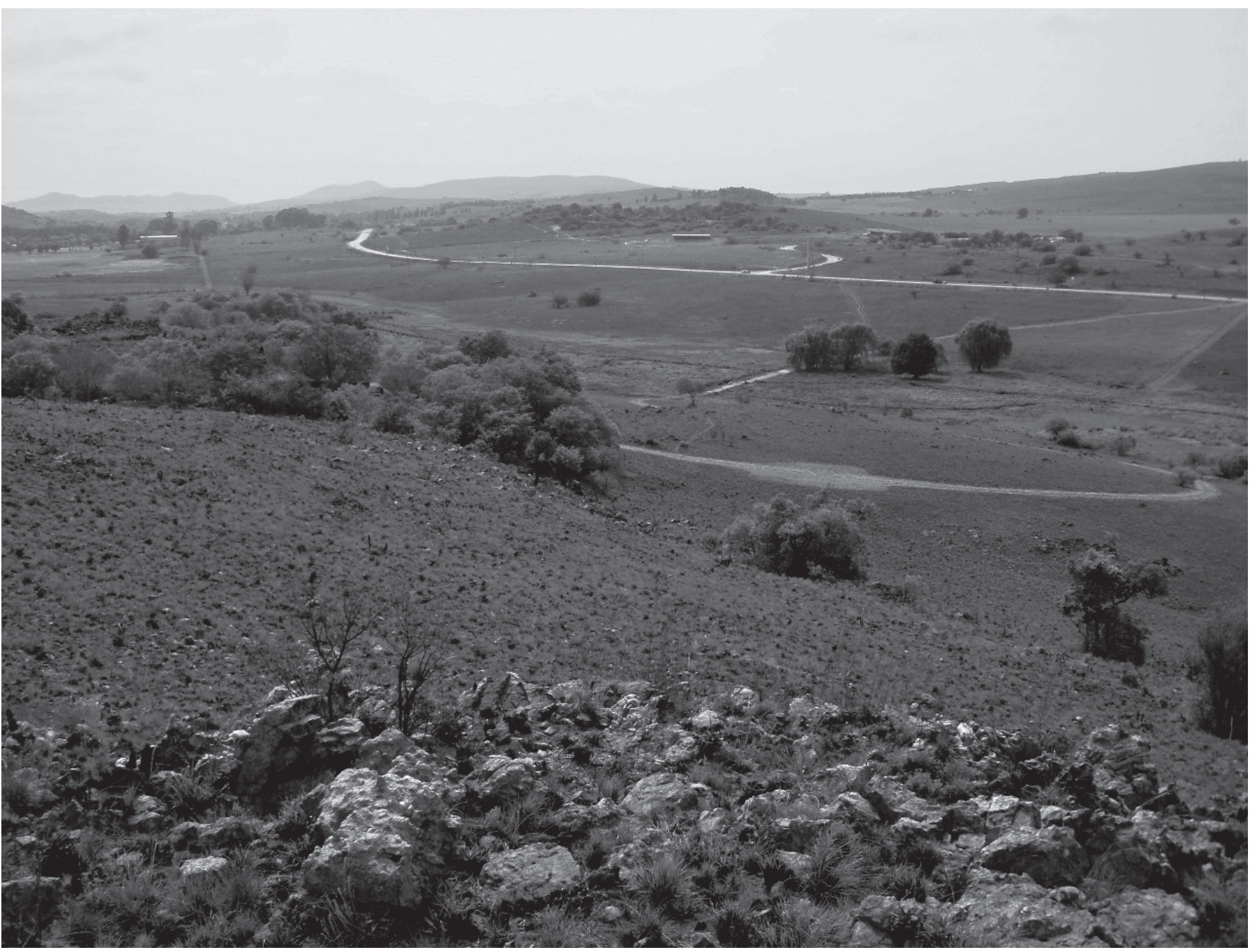

[Fig.1] Vue en direction du sud-est de la vallée de la Blauawbankspruit et du secteur de Sterkfontein depuis la colline de Swartkrans. 
l'ensemble des acteurs autour d'une stratégie commune combinant conservation, recherche archéologique et gestion. Enfin, la numérisation des plaques de verre et l'exploitation scientifique de ces anciennes photographies constituent une sauvegarde patrimoniale et un sauvetage de l'information exemplaires; ce recours aux sources anciennes est devenu indispensable pour comprendre le site de Paharpur et restituer en partie son authenticité archéologique.

Breuil J.-Y., Gill S., 2005, « Paharpur in the 20s-30s : a Pala period buddhist shrine and monastery documented by a collection of photographic plates ", in Proceedings of the Seventeenth International Conference of the European Association of South Asian Archaeologists, Bonn, 7-11 juillet 2003, p. 479-489.

Breuil J.-Y., GiLl S., 2007, « New researches on Paharpur Buddhist monastery (North Bengal) », in Hardy A. (ÉD.), The Temple in South Asia, vol. 2 of the Proceedings of the $18^{\text {th }}$ Conference of the European Association of South Asian Archaeologists, London 2005, The British Association for South Asian Studies, the British Academy, p. 127-138.

Dikshit K. N., 1938, Excavations at Paharpur, Bengal, Memoirs of the Archaeological Survey of India n ${ }^{\circ} 55$, Archaeological Survey of India, Delhi.

\section{Géoarchéologie dans le «Berceau de l'humanité » : Gauteng, Afrique du Sud}

\section{Laurent Bruxelles}

Inrap, UMR 5608 «Travaux et Recherches Archéologiques sur les Cultures, les Espaces et les Sociétés "; GAES, Université du Witwatersrand, Johannesburg

À environ $35 \mathrm{~km}$ au nord-ouest de Johannesburg, le long de la vallée de la Blauawbankspruit, un secteur incluant plusieurs cavités naturelles a été inscrit en 1999 sur la liste du Patrimoine mondial de l'Unesco sous le nom de « Berceau de l'humanité », terme attribué communément à la région depuis la fin des années 1930 après que de très nombreux restes d'hominidés y ont été retrouvés. Pourtant, ce n'est pas dans cette partie de l'Afrique que l'on trouve les fossiles d'hominidés les plus anciens. La particularité de l'endroit tient à leur densité. Ainsi, sur une surface d'à peine 25000 ha, ce sont plus d'un millier de vestiges d'hominidés anciens qui ont été mis au jour, soit un tiers des découvertes de ce type pour tout le continent africain. De plus, ces fossiles, dont l'âge s'étend de quatre à un millions d'années avant notre ère, couvrent une période chronologique clé : celle qui voit l'apparition des premiers hommes (genre Homo). Néanmoins, ce site exceptionnel se heurte à un gros problème de calage chronologique. En l'absence de datation directe sur les fossiles, celle des sédiments qui les contiennent a été obtenue par paléomagnétisme et par datation uranium/ plomb sur des planchers stalagmitiques. Or les résultats obtenus se sont révélés contradictoires et suscitent de vifs échanges par l'intermédiaire d'articles dans de prestigieuses revues internationales.

\section{Le contexte géologique : des dolomies}

karstifiées. L'ensemble de l'aire fossilifère se positionne dans la vallée de la Blauawbankspruit. Ce petit cours d'eau a entaillé une large vallée dans des dolomies vieilles de plus de 2,5 milliards d'années [Fig.1]. Ces dolomies, légèrement solubles dans l'eau, ont été profondément karstifiées au cours de la lente évolution morphologique de ce secteur. Ainsi, au moins depuis le Miocène (Martini et al., 2003), toute une série de cavités s'est développée par dissolution le long des fractures et des principales discontinuités de la roche. Il en résulte une grande densité de cavités qui dessinent un véritable labyrinthe en trois dimensions. Au fur et à mesure de l'encaissement du cours d'eau et de l'élargissement de la vallée, ces cavités ont été recoupées par la surface topographique. Le secteur est donc caractérisé par la présence de très nombreuses entrées de grottes qui correspondent à ces galeries partiellement décapitées. Beaucoup d'entre elles deviennent vite impénétrables, colmatées par des brèches et par d'imposants massifs stalagmitiques.

Ces brèches, aujourd'hui exposées à l'air libre, se sont initialement formées sous terre. Lors du démantèlement des cavités, des colluvions (argile, cailloutis, mais aussi restes végétaux et ossements) se sont accumulées à la base des entrées, constituant de vastes talus. Puis, les infiltrations d'eau, surchargées de calcite dissoute, ont peu à peu cimenté ces dépôts qui sont devenus de véritables brèches très indurées. Ainsi, chaque brèche en surface correspond à une ancienne galerie karstique recoupée par le versant.

L'approche géoarchéologique. En 2006, Ron J. Clarke et Kathleen Kuman, respectivement paléoanthropologue et archéologue à l'Université du Witwatersrand (Johannesburg), me contactent pour les aider à étudier ce karst, en particulier pour situer chronologiquement les séquences sédimentaires qui contiennent les fossiles. En tant que karstologue, c'est-à-dire spécialiste des paysages et des cavités en région calcaire (ou dolomitique), je suis a priori habitué à ce type de contexte parfois difficile à interpréter. Une première mission, financée par l'Université du Witwatersrand, est donc organisée en octobre 2007. Mais très vite, la visite des différents sites fossilifères (Sterkfontein, Swartkrans, Kromdraai, etc.) rend manifeste l'ampleur de la tâche. La stratigraphie est bien plus complexe que je ne pouvais l'imaginer et seul un véritable travail de fond permettra progressivement d'en maîtriser les grands traits. Pour répondre à la question de la datation, plusieurs approches complémentaires sont envisagées, mais à des échelles différentes, allant du paysage à la lame mince.

\section{Cartographie géomorphologique}

En premier lieu, il a semblé important de connaître l'histoire du paysage et des cavités qui s'y sont développées. Dès la mission de 2007, puis en 2008 et 2009 dans le cadre de la fouille du site de Kromdraai (direction J. Braga), nous avons commencé une cartographie des formes du paysage et des formations superficielles. Basée sur un travail de prospection minutieuse, cette approche permet d'identifier les principales 